\title{
Oversampling Technique For Peak To Average Power Ratio Measurement Of Discrete Time Ofdm Signal
}

\author{
Dr. Amandeep Singh Sappal ${ }^{1}$, Parneet Kaur ${ }^{2}$ \\ ${ }^{\prime}$ (ECE, University College of Engineering/ Punjabi University, India) \\ ${ }^{2}$ (ECE, University College of Engineering/ Punjabi University, India)
}

\begin{abstract}
When measured, the Peak to Average Power Ratio (PAPR) of the discrete Orthogonal Frequency Division Multiplexing (OFDM) signal comes out to be less as compared to its continuous counterpart. This happens due to non-consideration of some of the peaks while measuring PAPR of the discrete time OFDM signal. This paper presents oversampling technique for measuring the accurate value of PAPR of discrete time OFDM signal. Simulations have been performed on Chu sequence for different interpolation factor $L$.
\end{abstract}

Key words- Chu sequence, Interpolation, OFDM, Oversampling, PAPR.

\section{Introduction}

Compared with single-carrier systems, OFDM systems are known to have a high PAPR since in an OFDM system many subcarrier components are added via an IFFT operation. PAPR is the ratio between the maximum power and the average power of the complex pass band signal $\tilde{s}(t)$ [1], that is,

$$
\operatorname{PAPR}\left\{\tilde{s}_{I}(t)\right\}=\frac{\max \left|\tilde{s}_{I}(t)\right|^{2}}{E\left\{\left|\tilde{s}_{I}(t)\right|^{2}\right\}}
$$

The PAPR defined in (1) deals with the pass band signal with a carrier frequency of $f_{c}$ in the continuous time domain. As $f_{c}$ generally is much higher than $1 / T_{s}$, a continuous time baseband OFDM signal $x(t)$ with the symbol period $T_{s}$ and the corresponding pass band signal $\tilde{x}(t)$ with the carrier frequency $f_{c}$ have almost the same PAPR [2]. But the PAPR for the discrete-time baseband signal ${ }^{x[n]}$ may not be the same as that for the continuous-time baseband signal ${ }^{x(t)}$. Indeed, as $x[n]$ may not have all the peaks of $x(t)$, the PAPR for

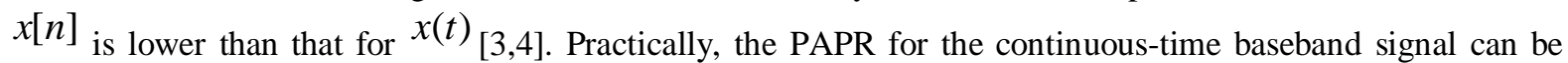
measured only after implementing the actual hardware, including digital-to-analog convertor (DAC). It implies that measurement of the PAPR for the continuous-time baseband signal is not straightforward. For that reason, there must be some means of estimating the PAPR from the discrete-time signal ${ }^{x[n]}$.Oversampling is on e of the methods using which actual value of PAPR for the discrete time signal $x[n]$ can be calculated [3]. This paper presents oversampling technique to calculate the PAPR of discrete time signal $x[n]$. The paper has been organized as follows: Section I is introduction, Section II presents the method of oversampling for calculating PAPR, Section III presents the Simulation results obtained using MATLAB and Section IV provides with conclusions.

\section{Oversampling Technique for measurement of PAPR}

$x[n]$ can show almost the same PAPR as $x(t)$ if it is L-times interpolated (oversampled) where $L \geq 4$

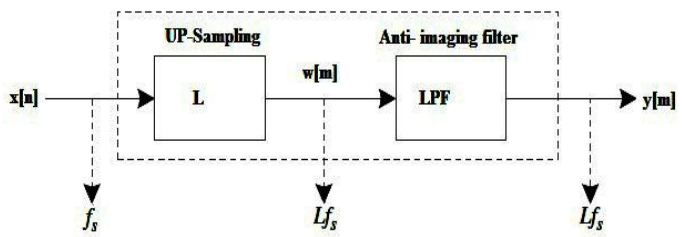

Fig.1 Block Diagram of L-times interpolator 
The block diagram of interpolator with a factor of $\mathrm{L}$ is shown in Fig.1 which inserts (L-1) zeros between the samples of $x[n]$ to yield $w[m]$ as follows:

$w[m]=\left\{\begin{array}{c}x[m / L], \text { for } m=0, \pm L, \pm 2 L \ldots \ldots \\ 0, \text { elsewhere }\end{array}\right.$

To construct the L-times-interpolated version of $x[n]$ from $w[m]$ a low pass filter (LPF) is used. For the LPF with an impulse response of $h[m]$, the L-times-interpolated output $y[m]$ can be represented as

$$
y[m]=\sum_{k=-\infty}^{\infty} h[k] w[m-k]
$$

(3)

Figures (2) and (3) illustrate the signals and their spectra appearing in the oversampling process with a sampling frequency of $2 \mathrm{kHz}$ to yield a result of interpolation with $\mathrm{L}=4$. As per these figures, the IFFT output signal $x[n]$ can be expressed in terms of the L-times interpolated version as

$x^{\prime}[m]=\frac{1}{\sqrt{L . N}} \sum_{k=0}^{L . N-1} X^{\prime}[k] \cdot e^{j^{2 \pi m \Delta \Delta k} / L . N}, m=0,1, \ldots . . N L-1$

With

$$
X^{\prime}[k]=\left\{\begin{array}{c}
X[k], \text { for } 0 \leq k \leq N / 2 \text { and } N L-N / 2<k<N L \\
0, \text { elsewhere }
\end{array}\right.
$$

where $\mathrm{N}, \Delta f$, and $X[k]$ denote the FFT size, the subcarrier spacing and the complex symbol
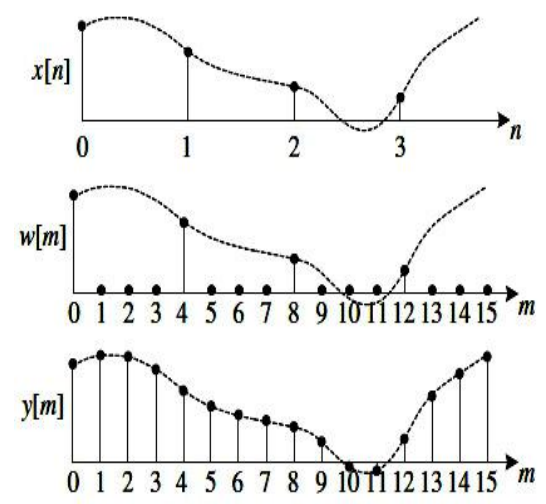

Fig. 2 Interpolation with $\mathrm{L}=4$ in the time domain [1]
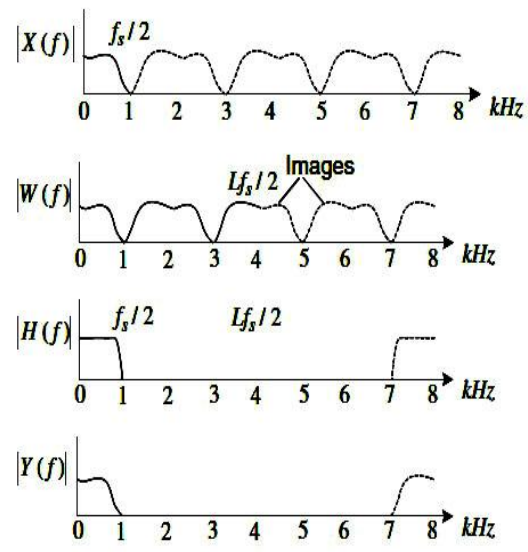

Fig.3 Interpolation with $\mathrm{L}=4$ in the frequency domain [1]

Www.iosrjournals.org 
carried over a subcarrier k, respectively. For such an L-times-interpolated signal, the PAPR will be redefined as

$P A P R=\frac{\operatorname{mo,1,\ldots ,NL}_{m}\left|x^{\prime}[m]\right|^{2}}{E\left\{\left|x^{\prime}[m]\right|^{2}\right\}}$

In order to see the effect of oversampling or interpolation on PAPR, let us consider the PAPRs of the specific sequences, for example, Chu sequence. The Chu sequence is defined in $[5,6]$ as

$X_{i}(k)= \begin{cases}e^{j \frac{\pi}{N} i^{2} k} & \text { if } \mathrm{N} \text { is even } \\ e^{j \frac{\pi}{N}(i+1) k} & \text { if } \mathrm{N} \text { is odd }\end{cases}$

\section{Implementation of Oversampling Technique for chu sequence}

Simulation results for the effect of oversampling on the measurement of PAPR are obtained by using MATLAB. Table 1 shows the value of PAPR with and without oversampling with $\mathrm{N}=16$ point IFFT of chu sequence and varying number of $\mathrm{L}$.

Table.1 PAPR for $\mathrm{N}=16$ and varying $L$

\begin{tabular}{|l|l|l|}
\hline Value of L & $\begin{array}{l}\text { Without } \\
\text { oversampling }\end{array}$ & $\begin{array}{l}\text { With } \\
\text { Oversampling }\end{array}$ \\
\hline $\mathrm{L}=3$ & 0.0000 & 3.8872 \\
\hline $\mathrm{L}=4$ & 0.0000 & 4.2714 \\
\hline $\mathrm{L}=5$ & 0.0000 & 4.1551 \\
\hline $\mathrm{L}=6$ & 0.0000 & 4.2714 \\
\hline
\end{tabular}

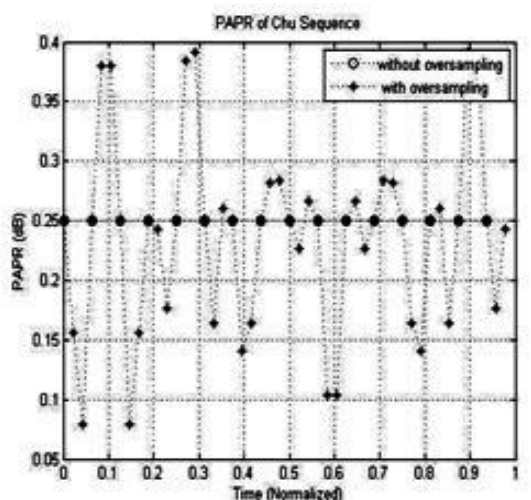

(a) $\mathrm{L}=3$

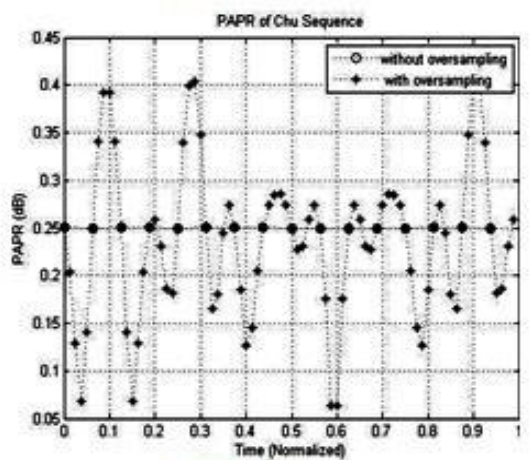

(c) $\mathrm{L}=5$

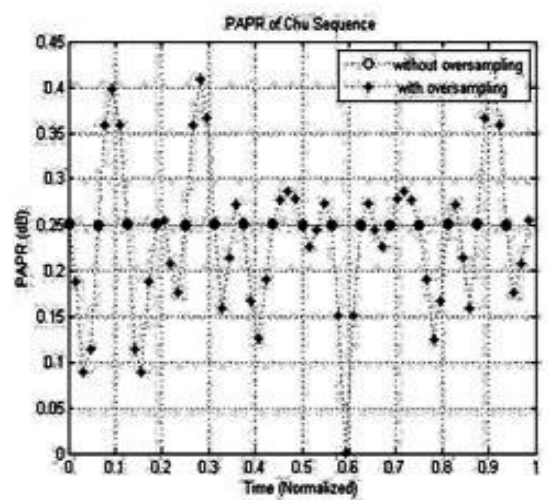

(b) $\mathrm{L}=4$

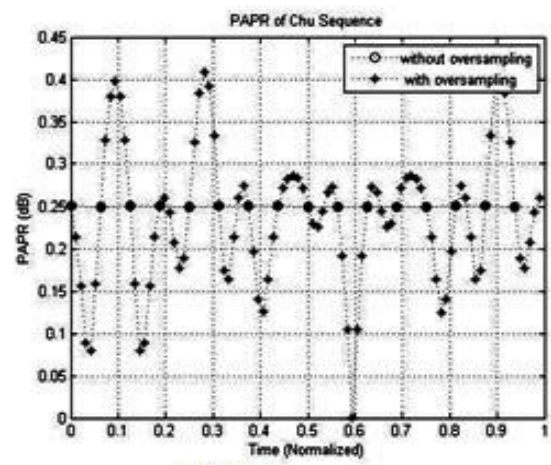

(d L $=6$

Fig. 4 PAPR characteristics of chu sequence in the timed domain with $\mathrm{N}=16$ and varying $\mathrm{L}$ 
By using oversampling technique the value of PAPR has been calculated as 4.271 , which is very close to the actual value.

\section{Conclusions}

The PAPR for the discrete time baseband signal can't be measured accurately by using the techniques used for measurement of PAPR of continuous time signal. Oversampling is a technique, which can be used to calculate the more accurate value of PAPR of discrete time signal. Results show that measured value of PAPR of Discrete time OFDM signal comes out to be same as the actual value of 4.27 when the value of $\mathrm{L}$ has been taken $\geq 4$.

\section{REFRENCES}

[1]. Yong soo cho, Jaekwon Kim, Won young Yang and Chung G. Kang, MIMO-OFDM wireless communication with MATLAB. John Wiley \& Sons. (2010).

[2]. Ochiai, H. and Imai, K., The distribution of the peak-to-average power ratio in OFDM signals. IEEE Trans. Commun., 49(2), 282289 (2001).

[3]. Han, S.H. and Lee, J.H., An overview of peak-to-average power ratio reduction techniques for multicarrier transmission. IEEE Wireless Communication, 12(2), 56-65 (2005).

[4]. Ochiai, H. and Imai, K., Clipping for peak power reduction of OFDM signals. IEEE GTC, vol. 2, pp. 731-735 (2000).

[5]. Chu, D.C., Polyphase codes with periodic correlation properties. IEEE Trans. Info. Theory, 18(4), 531-532 (1972).

[6]. Luke, H.D., Schotten, H.D., and Mahram, H.H., Binary and quadriphase sequences with optimal autocorrelation properties. IEEE Trans. Info. Theory, 49(12), 3271-3282 (2003). 